\title{
L'innocuité d'une éventuelle vaccination contre le SIDA en question !
}

L'une des voies explorées actuellement pour la vaccination contre le SIDA est l'utilisation d'un virus de la vaccine recombiné par l'insertion du gène codant pour la glycoprotéine de surface d'HIV. Nous avons relaté ici combien cette stratégie restait d'efficacité incertaine puisque les malades atteints du SIDA ont des anticorps dirigés contre cette protéine... et n'en sont pas moins malades. Par ailleurs, la variabilité génétique du virus risque de faire apparaître des variants de cette protéine que ne reconnaîtraient plus les anticorps vaccinaux. Deux articles récents aggravent les doutes que l'on peut légitimement et malheureusement avoir quant à la possibilité d'utiliser un tel système [1,2] : l'expression de la glycoprotéine de sur- face d'HIV des cellules en culture infectées par un virus vaccinal recombiné peut suffire à conduire au phénomène de fusion, formation de syncytium et mort cellulaire caractéristique de la maladie. La fusion se fait entre des cellules exprimant à leur surface l'antigène de différenciation CD4 et implique une interaction très spécifique entre cet antigène et la glycoprotéine d'HIV. Certes, la proportion des cellules circulantes possédant l'antigène CD4 est faible chez l'homme, et, une fois la réaction immune déclenchée par vaccination à l'aide du virus vaccinal recombiné, les anticorps produits devraient limiter l'expression de la glycoprotéine en s'opposant à la réplication du virus vaccinal. Il est clair cependant que c'est avec d'infinies réticences et après de multiples contrôles que l'on se résoudrait à utiliser un vaccin potentiellement pathogène. Il semble a priori plus probable que la glycoprotéine naturelle d'HIV devra être remplacée, à des fins vaccinales, par des dérivés obtenus par mutation supprimant l'effet pathogène de cette protéine tout en lui conservant ses propriétés antigéniques. $\mathbf{A} . \mathbf{K}$.

1. Sodroski J, Goh WC, Rosen C, Campbell $\mathrm{K}$, Haseltine WA. Role of the HTLV-III/LAV envelope in syncytium formation and cytopathicity. Nature $1986 ; 322: 470-4$.

2. Lifson JD, Feinberg MB, Reyes GR, et al. Induction of CD4-dependent cell fusion by the HTLV-III/LAV envelope glycoprotein. Nature $1986 ; 323$ : 725-7.

\section{Espoirs dans le traitement des blessures du système nerveux central}

Lors d'un symposium récent à Luxembourg [1] et dont certains résultats sont rapportés par Sabel et Stein [2], sont apparus de nouveaux espoirs pour la thérapeutique médicale des blessures du cerveau et de la moelle épinière. Ces perspectives sont d'autant plus actuelles qu'elles mettent en jeu le Facteur de croissance du nerf (NGF) dont la découverte vient de valoir le prix Nobel de médecine à Rita Levi-Montalcini. Connu depuis trente ans, il manquait à ce facteur les lettres de noblesse que confere une action thérapeutique.

L'idée fondamentale s'étaye sur une double constatation : dès les premières minutes qui suivent une lésion traumatique du système nerveux central, le tissu resté indemne commence à dégénérer, par suite d'un milieu biochimique $m / s n^{\circ} 2$ ool. 3, févier 87 défavorable; en revanche, s'il s'avère possible de sauver, ne fûtce qu'une proportion limitée, des axones ventraux (de la moelle par exemple), la locomotion a de fortes chances d'être préservée ; il est donc capital d'empêcher les neurones survivants d'être détruits. Sur quelles bases pourrait-on y parvenir?

La détérioration secondaire est due, au moins en partie, à des métabolites des acides gras qu'il faudrait neutraliser, à l'élévation des prostaglandines et du thromboxane. Les conséquences de l'ischémie peuvent être en outre réduites par des antagonistes de la sérotonine; et des agonistes des catécholamines ont pu améliorer le fonctionnement de neurones survivants.

Les méthodes que nous venons de citer ont comme unique objectif d'aider les neurones épargnés à survivre. Est-il possible, en plus, de stimuler la régénération? On se tourne, à cette fin, vers les cellules gliales, et notamment les astrocytes qui peuvent proliférer après un traumatisme et qui sont en train de perdre leur mauvaise réputation, accusés qu'ils étaient de dresser une clôture autour de la lésion. Il semblerait qu'ils libèrent en fait des facteurs trophiques capables de stimuler la croissance de neurones. Cependant, cet effet trophique ne se fait sentir qu'avec retard, d'où l'importance de découvrir des neurotrophiques qui, employés à une phase précoce, contrebalanceraient les effets neurotoxiques des métabolites des acides gras et de certains acides aminés.

Deux agents pharmacologiques biologiques ont fait l'objet d'une

(n) 
étude exhaustive. L'un est un ganglioside appelé $\mathrm{GM}_{1}$, qui serait un potentiateur de facteurs trophiques endogènes. L'autre est le NGF, dont les propriétés autorisent beaucoup d'espoirs. Jusqu'à une date récente, le NGF était considéré comme agissant sélectivement sur les neurones sympathiques et sensitifs du système nerveux périphérique. Hefti [3] a montré qu'il pouvait, après section de l'axe septum-hippocampe chez le rat, avoir une action salvatrice sur les neurones cholinergiques. Après transsection bilatérale, l'injection intraventriculaire de NGF atténue fortement la perte de performances dans les tests de comportement du rat. A l'étape actuelle des connaissances toutefois, le NGF, comme les gangliosides, semble surtout permettre la survie des neurones épargnés par la lésion, plus que la régénération de nouveaux neurones. Ce serait déjà, par rapport à la situation antérieure, un progrès considérable.

Le même auteur [3] suggère enfin une application possible du NGF à la maladie d'Alzheimer, au cours de laquelle la perte de neurones cholinergiques reste l'anomalie biochimique la mieux caractérisée. Cette proposition reste valable bien que Goedert et al. [4] n'aient pas trouvé de diminution du taux de l'ARN messager du NGF dans le cortex de sujets atteints de maladie d'Alzheimer, car il pourrait exister chez certains sujets âgés une baisse de la sensibilité des cellules cérébrales au NGF.

J.-C. D.

1. Pharmacological approaches to the treatment of brain and spinal cord injury. Walferdange, Luxembourg, 7-11 juillet 1986.

2. Sabel BA, Stein DG. Pharmacological treatment of central nervous system injury. Nature $1986 ; 323$ : 493.

3. Hefti F. Nerve growth factor promotes survival of septal cholinergic neurones after fimbrial transections. J Neurosci $1986 ; 6$ : 2155-62. 4. Goedert $M$, Fine A, Hunt SP, Ulrich A a al. Nerve growth factor mRNA in peripheral and central rat tissues and in the human central nervous system : lesion effects and levels in Alzheimer disease. Mol Brain Res $1986 ; 1: 85-92$.

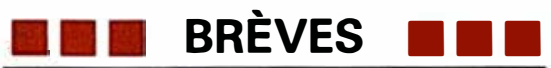

Le SIDA est-il, à terme, inéluctable chez les sujets infectés par le virus HIV? La première étude prospective à long terme sur le devenir des sujets infectés par le virus du SIDA (HIV) vient d'être publiée en Allemagne. Elle est basée sur l'observation de 543 personnes, principalement les partenaires sexuels de malades atteints. Ces résultats sont proprement terrifiants puisqu'ils indiquent que, sur une période de 7 ans, les $3 / 4$ de ces sujets entrent dans la phase terminale du SIDA. Ce que de nombreux scientifiques virologues, médecins et épidémiologistes, pressentaient depuis quelques temps pourrait être vrai : le SIDA et la mort pourraient bien être, en l'absence d'un traitement découvert d'ici là, le terme quasi inéluctable des infections par HIV. Oui, vraiment, depuis les grandes épidémies du Moyen Âge, nulle maladie infectieuse n'avait constitué un pareil danger pour l'humanité.

Brodt HR, et al. Dtsch Med Wochenschr 1986 ; 111 : 1175-80.

L'analyse des $\beta$ thalassémies (37 mutations reconnues à ce jour) montre qu'une même mutation a pu apparaître indépendamment à plusieurs reprises, y compris dans des ethnies différentes. Le dernier exemple porte sur une mutation située dans la zone régulatrice (TATA box) : le remplacement d'une adénine par une guanine en position - 29 provoque l'apparition d'une $\beta$ + thalassémie dans des familles chinoises et chez des Noirs des USA. Ces observations sont en accord avec celles que rapporte $\mathrm{D}$. Labie dans la drépanocytose $\left(\mathrm{m} / \mathrm{s} n^{\circ} 1\right.$, vol 3, p. 54)

Huang S. et al. Hum Genet 1986 ; $74: 162-4$.

\section{La nature de l'acide}

La durée de vie des protéines (mesurée par leur " demi-vie ") est extraordinairement variable dans un même organisme, allant de plusieurs jours à moins d'une minute. On ignore les mécanismes de ces différences, et on les imagine complexes. Une première réponse vient d'être fournie par le groupe de Varshavsky (Cambridge, USA) ; elle est d'une simplicité inattendue $[1,2]$.

Les recherches qui ont abouti à ce résultat n'avaient nullement pour objet la longévité des protéines. La question posée tenait au rôle de l'ubiquitine dans la dégradation des protéines. L'ubiquitine $\left(\mathrm{m} / \mathrm{s} n^{\circ} 5\right.$, vol. 2, p. 283) se lie par son $\mathrm{COOH}$ terminal à des groupes $\epsilon \mathrm{NH} 2$ de résidus lysine des protéines, en formant une structure branchée. Il est possible mais non démontré que l'ubiquitine se fixe également au groupement aminé terminal. Varshavsky et al. ont construit un gène chimérique en liant de l'ubiquitine de levure à de la $B$ galactosidase d' $E$. Coli, et l'ont fait exprimer dans le colibacille et la levure. L'E. Coli exprime le gène chimérique dans son entier, car les bactéries ne possèdent ni ubiquitine ni enzymes pour la cliver. Dans la levure, on retrouve uniquement la B galactosidase libre, sa liaison avec l'ubiquitine étant immédiatement scindée. L'objectif initial - rôle de l'ubiquitine dans la dégradation des protéines - s'avérait hors de portée.

L'acide aminé $\mathrm{N}$-terminal de la $\beta$ galactosidase est la méthionine. Pouvait-on tourner la difficulté et obtenir un produit stable ubiquitine-gal en remplaçant la méthionine par d'autres acides aminés ? Par mutagenèse dirigée sur le codon ATG initiateur de la méthionine, Bachmair parvint à remplacer la méthionine initiale par 15 acides aminés différents. A l'exception de la proline, tous ces. mutants se débarrassaient de l'ubiquitine et libéraient de la B 